\title{
257. A Table of Fundamental Units of Purely Cubic Fields
}

\author{
By Hideo WADA \\ Department of Mathematics, University of Tokyo \\ (Comm. by Kunihiko KodaIRA, M. J. A., Dec. 12, 1970)
}

The following table shows the fundamental unit of the real cubic field $Q(\sqrt[3]{m})$, for $2 \leqq m \leqq 250$. In the field $Q(\sqrt[3]{m})$, there is only one fundamental unit $\varepsilon(>1)$, and any unit in $Q(\sqrt[3]{m})$ can be expressed as $\pm \varepsilon^{n}(n \in \boldsymbol{Z})$ by Dirichlet theorem.

In 1892, A. A. Markov published the table of a unit of $Q(\sqrt[3]{m})$, for $2 \leqq m \leqq 70$. But in his table, two units were not fundamental units $(m=28,55)$. In 1896, G. F. Voronoi gave an algorithm of finding fundamental units of fields $Q(\sqrt[3]{m})$. It was based on a generalization of the algorithm of continued fractions. In 1955, K. K. Billebič [1] gave a method of finding a system of fundamental units of any algebraic number field.

In 1900, R. Dedekind [3] calculated ideal class numbers of $Q(\sqrt[3]{m})$ by analytic method using Markov's table. If the author's table is used, ideal class numbers of $Q(\sqrt[3]{m}), 2 \leqq m \leqq 250$, can be calculated. Recently the author has found an algorithm of calculating the structure of ideal class group of any finite algebraic number field by geometrical method. This method will be published later.

For computing this table, the auther used the electoronic computer TOSBAC-3300 installed in the Department of Mathematics University of Tokyo. This calculation required five hours computer time.

Table

\begin{tabular}{r|l}
\hline$m$ & \multicolumn{2}{|c}{ Fundamental unit $(>1), \alpha=\sqrt[3]{m}$} \\
\hline 2 & $1+\alpha+\alpha^{2}$ \\
3 & $4+3 \alpha+2 \alpha^{2}$ \\
5 & $41+24 \alpha+14 \alpha^{2}$ \\
6 & $109+60 \alpha+33 \alpha^{2}$ \\
7 & $4+2 \alpha+\alpha^{2}$ \\
10 & $\left(23+11 \alpha+5 \alpha^{2}\right) / 3$ \\
11 & $89+40 \alpha+18 \alpha^{2}$ \\
12 & $\left(110+48 \alpha+21 \alpha^{2}\right) / 2$ \\
13 & $94+40 \alpha+17 \alpha^{2}$ \\
14 & $29+12 \alpha+5 \alpha^{2}$ \\
15 & $5401+2190 \alpha+888 \alpha^{2}$ \\
17 & $324+126 \alpha+49 \alpha^{2}$ \\
19 & $\left(14+5 \alpha+2 \alpha^{2}\right) / 3$
\end{tabular}


Table (Continued)

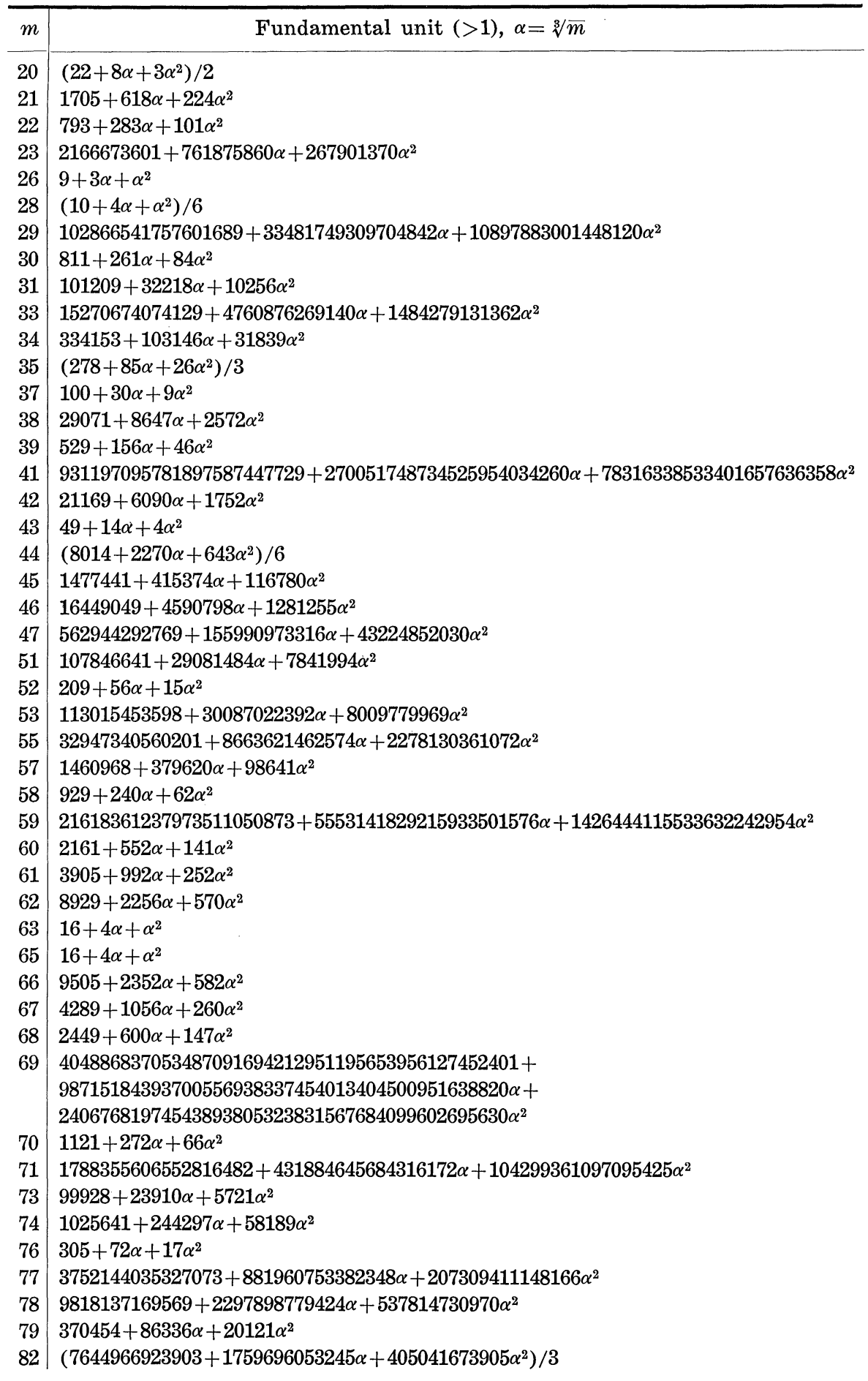


Table (Continued)

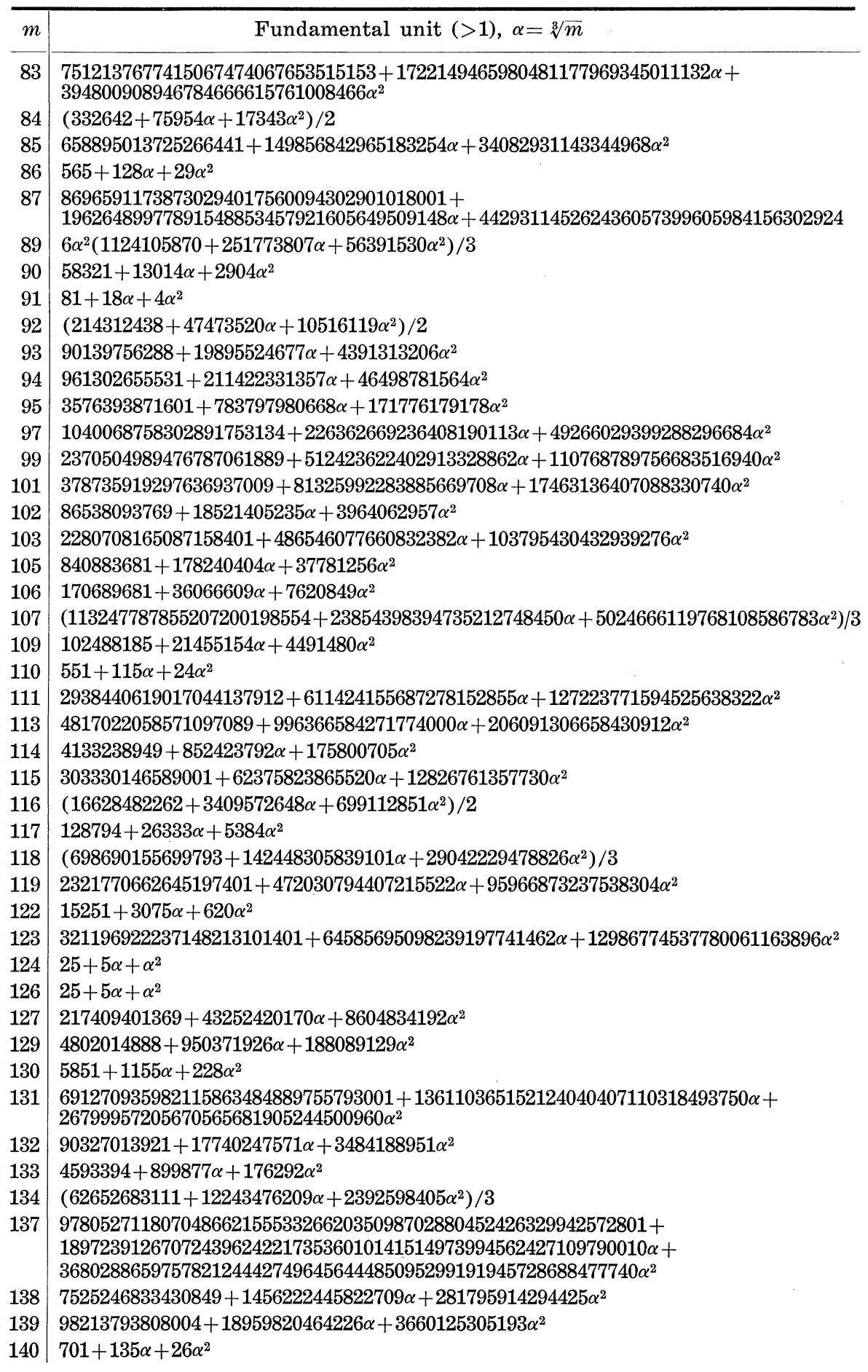


Table (Continued)

\begin{tabular}{|c|c|}
\hline$m$ & Fundamental unit $(>1), \alpha=\sqrt[3]{m}$ \\
\hline 141 & $90941859649+17472596988 \alpha+3356998050 \alpha^{2}$ \\
\hline 142 & $552019321+105809541 \alpha+20281281 \alpha^{2}$ \\
\hline 143 & $\left(7305401975+1397007610 \alpha+267148922 \alpha^{2}\right) / 3$ \\
\hline 145 & $\begin{array}{l}4601120912488201407747405990121+875805454131464787106374958632 \alpha+ \\
166706158798079221119438870726 \alpha^{2}\end{array}$ \\
\hline 146 & $658031496221030890375+124967110857372704197 \alpha+23732570379569259472 \alpha^{2}$ \\
\hline 148 & $1878269+355089 \alpha+67130 \alpha^{2}$ \\
\hline 149 & $\begin{array}{l}859699668151966502259032173489+162162837995150600262255107252 \alpha+ \\
30588340324906413785889988460 \alpha^{2}\end{array}$ \\
\hline 150 & $\left(1355+255 \alpha+48 \alpha^{2}\right) / 5$ \\
\hline 151 & $58886554777961+11058354219854 \alpha+2076657371328 \alpha^{2}$ \\
\hline 153 & $1888+353 \alpha+66 \alpha^{2}$ \\
\hline 154 & $\left(2155235855+402088109 \alpha+75014921 \alpha^{2}\right) / 3$ \\
\hline 155 & $807243088561096721+150277433472159724 \alpha+27975844365832758 \alpha^{2}$ \\
\hline 156 & $\left(3789756830+703995384 \alpha+130776069 \alpha^{2}\right) / 2$ \\
\hline 157 & $\begin{array}{l}4012030799617318079361745+743699873398009028217598 \alpha+ \\
137857740709510585418372 \alpha^{2}\end{array}$ \\
\hline 158 & $89455153351649+16547015316624 \alpha+3060793097210 \alpha^{2}$ \\
\hline 159 & $\begin{array}{l}830240612335926573133077433+153251569942463356234249896 \alpha+ \\
28288237579405435437820554 \alpha^{2}\end{array}$ \\
\hline 161 & $39275546844846+7219607961969 \alpha+1327104096868 \alpha^{2}$ \\
\hline 163 & $512936998354+93900554724 \alpha+17189858025 \alpha^{2}$ \\
\hline 164 & $162361+29662 \alpha+5419 \alpha^{2}$ \\
\hline 165 & $130681+23826 \alpha+4344 \alpha^{2}$ \\
\hline 166 & $1767569+321618 \alpha+58520 \alpha^{2}$ \\
\hline 167 & $\begin{array}{l}2070166880101044205370118762758521169120826289178053244249389075109893279095936 \\
78542437176000329+ \\
3759238378430387052058014955399294460976175786237429132766935800446953722774928 \\
4245814885920536 \alpha+ \\
6826441540391252694667966480938649885916157400845126642231195944528075516296200 \\
936950484198110 \alpha^{2}\end{array}$ \\
\hline 170 & $175601374201+31698954351 \alpha+5722185897 \alpha^{2}$ \\
\hline 171 & $6100+1099 \alpha+198 \alpha^{2}$ \\
\hline 172 & $\left(117310+21094 \alpha+3793 \alpha^{2}\right) / 6$ \\
\hline 173 & $\begin{array}{l}1298828639084088159278484528360730410533356984157704652806743334753+ \\
233096895019168539395020385397325352385828994785662845633023003262 \alpha+ \\
41833203266824178774723154719985171566527663453915879687183651996 \alpha^{2}\end{array}$ \\
\hline 174 & $235444969+42173502 \alpha+7554225 \alpha^{2}$ \\
\hline 175 & $\left(378005+67580 \alpha+12082 \alpha^{2}\right) / 5$ \\
\hline 177 & $\begin{array}{l}1529999819870129383038273618656214656864166186711884183651770943819074875333937 \\
7449993+ \\
2725002829532353198471015944634228563892804306074581570673840009815879882246402 \\
575848 \alpha+ \\
4853360323656534864543756607695913090288163008411354585576914770631365148042008 \\
51406 \alpha^{2}\end{array}$ \\
\hline 178 & $4218014114104368961+749839005206339784 \alpha+133299348584142966 \alpha^{2}$ \\
\hline 179 & $\begin{array}{l}1565882142419862384470856245195930911194+ \\
277848502886388600942247003648177715976 \alpha+ \\
49301150108848866524199807763681342081 \alpha^{2}\end{array}$ \\
\hline 181 & $\left(16966724329842756278+2999428257712544915 \alpha+530247895720239530 \alpha^{2}\right) / 3$ \\
\hline 182 & $289+51 \alpha+9 \alpha^{2}$ \\
\hline 183 & $\begin{array}{l}4233504031039334302315890452620079552467606161001+ \\
745675054088764429586069727215344121157283327710 \alpha+ \\
131340677182200506036311403064866045202757322400 \alpha^{2}\end{array}$ \\
\hline
\end{tabular}


Table (Continued)

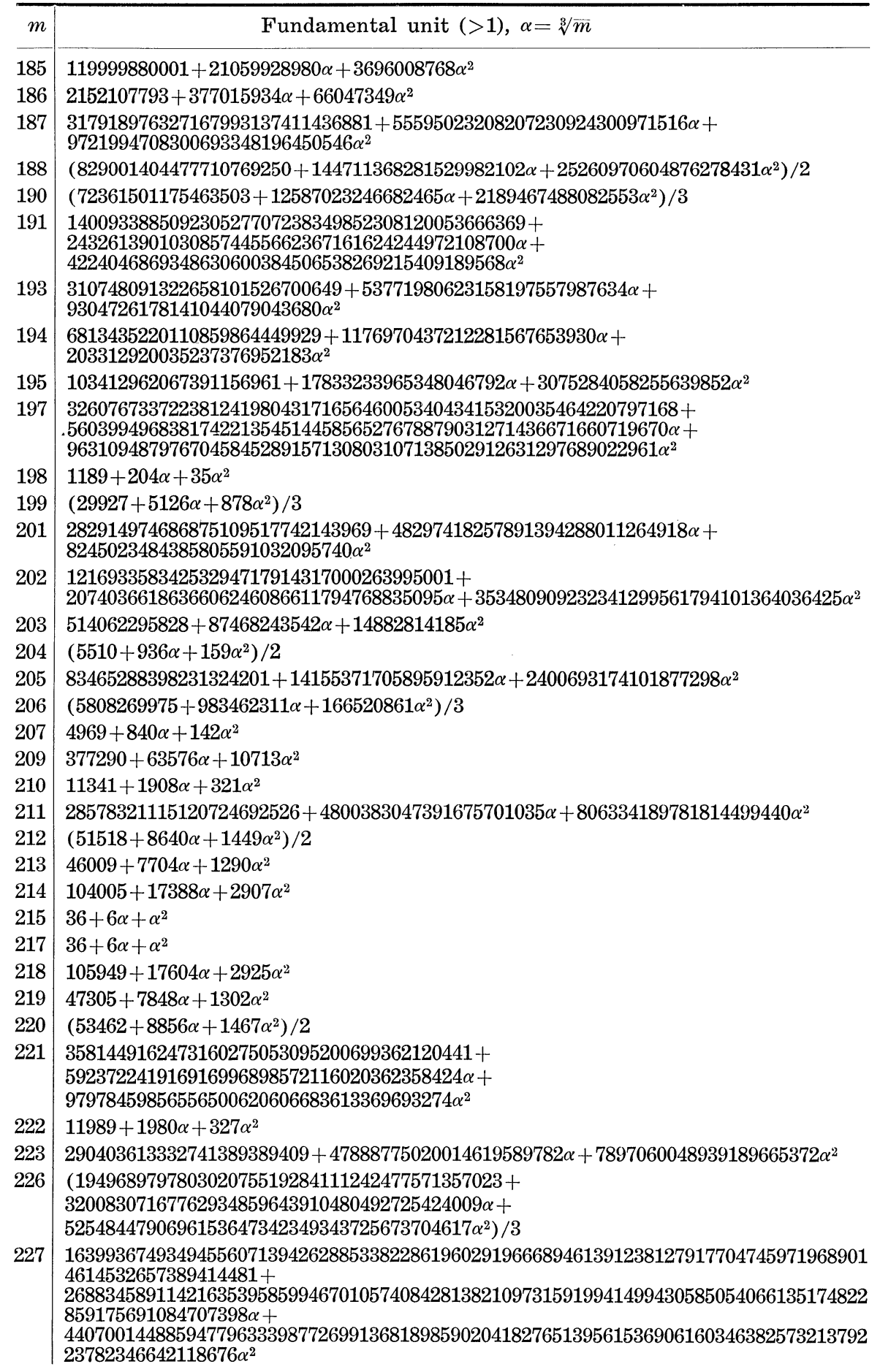


Table (Continued)

\begin{tabular}{|c|c|}
\hline$m$ & Fundamental unit $(>1), \alpha=\sqrt[3]{m}$ \\
\hline 228 & $\left(6158+1008 \alpha+165 \alpha^{2}\right) / 2$ \\
\hline 229 & $45665+7464 \alpha+1220 \alpha^{2}$ \\
\hline 230 & $23809996981+3886124664 \alpha+634269921 \alpha^{2}$ \\
\hline 231 & $44914+7320 \alpha+1193 \alpha^{2}$ \\
\hline 233 & $\left(386519830942502+62813521316866 \alpha+10207855184567 \alpha^{2}\right) / 3$ \\
\hline 234 & $1405+228 \alpha+37 \alpha^{2}$ \\
\hline 235 & $\left(229338383+37163858 \alpha+6022334 \alpha^{2}\right) / 3$ \\
\hline 236 & $13901581+2249539 \alpha+364018 \alpha^{2}$ \\
\hline 237 & $\begin{array}{l}16256982906342583000470053231327892613176118654+ \\
2626982423778989162357847356780028710739354756 \alpha+ \\
424496765150151362812472543258871517156352249 \alpha^{2}\end{array}$ \\
\hline 238 & $10624321+1714387 \alpha+276641 \alpha^{2}$ \\
\hline 239 & $\begin{array}{l}1297210666070701695128424020205447169311981562637931767629831831847637335154224 \\
6445449784249440932371355990207548750021841241680343074292776472564418698366859 \\
864906567294925737472124385185+ \\
2090312466171118485744217392622240864356232614474027745711317658247883084085566 \\
3855314750825580355585325403316298297126656439845907573193746257843228824316927 \\
96407651741158025184644414948 \alpha+ \\
3368308880365186741611096692335036277799365038863631233365617782384319138983248 \\
0710694770736125720916800646155415445039031513414259559821610417463203990814605 \\
5728760523786803598551081222 \alpha^{2}\end{array}$ \\
\hline 241 & $\begin{array}{l}1010320852597992917321596786624+162350502016341453001703057210 \alpha+ \\
26088430657627754711967317353 \alpha^{2}\end{array}$ \\
\hline 244 & $\left(1035104946+165648602 \alpha+26508867 \alpha^{2}\right) / 2$ \\
\hline 246 & $\begin{array}{l}1278056793936886730798199478071713668598271054581873251+ \\
203972577680300684983807733369748320549630887754141625 \alpha+ \\
32553179673172516899058456925099480370680361875515440 \alpha^{2}\end{array}$ \\
\hline 247 & $3106162477570+495060775756 \alpha+78902882081 \alpha^{2}$ \\
\hline 249 & $\begin{array}{l}9115694171340598924880665761574035823569796182762397129+ \\
1448960781078626614634391426899068579521519507254051502 \alpha+ \\
230315684756591912246482085511242733348857722291840680 \alpha^{2}\end{array}$ \\
\hline
\end{tabular}

\section{References}

[1] K. K. Billebič: On the units of algebraic fields of third and fourth degree. Mat. Sb., 123-136 (1956).

[ 2 ] H. Cohn: A numerical study of Dedekind's cubic class number formula. J. Res. Nat. Bur. Standards, 59, 265-271 (1957).

[ 3 ] R. Dedekind: Über die Anzahl der Idealklassen in reinen kubischen Zahlkörpern. J. Reine Angew. Math., 121, 40-123 (1900).

[4] B. N. Delone and D. K. Faddeev: The Theory of Irrationalities of the Third Degree. Trudy Mat. Inst. Steklov. (1940), Trans. Amer. Math. Soc. Vol. 10 (1955). 